\title{
Characteristics of Capacity Coupled Discharge in Atmospheric Pressure Air
}

\author{
Tadahiro Sasaki Student Member (Iwate University) Reina Omukai Non-member (Tohoku Electric Power Co.,Inc) \\ Seiji Mukaigawa Member (Iwate University) \\ Koichi Takaki Member (Iwate University) \\ Tamiya Fujiwara Member (Iwate University) \\ Hiroshi Mase Member (Ibaraki University) \\ Noriyoshi Sato Membe (Tohoku University)
}

Keywords : capacity-coupled multi-discharge, dielectric barrier discharge, power, streamer

Research on atmospheric pressure nonthermal plasmas is motivated by various industrial applications. Capacity-coupled multi-discharge (CCMD) has been proposed as a large-scale, atmospheric pressure, dense plasma source. The discharge gaps in the CCMD consist of a common electrode and a number of compact electrodes which are directly coupled with small capacitors for quenching the discharge. This paper describes characteristics of capacity coupled discharge in atmospheric pressure air with forcusing influence of input power into the discharge on gap length of point-to-plane electrode configuration.

Figure 1 shows the Vzexperimental setup. The needle electrode was connected to the quenching capacitor. A damping resistor $R_{0}$ was employed to reduce the inductance and capacitance (LC) resonant oscillation due to low impedance of the discharge plasma after breakdown. A typical value of the resistance $R_{0}$ was $50 \mathrm{k} \Omega$. The gap length was changed from 1 to $5 \mathrm{~mm}$. The discharge gap was placed in ambient air. We used mainly the $V-Q$ Lissajous diagram to measure the deposited energy. The electric charge was measured using a $0.05 \mu \mathrm{F}$ capacitor $C_{L}$.

Figure 2 shows the input power to the plasma as a function of the applied voltage for two different gap lengths at $9.4 \mathrm{pF}$ of quenching capacitor capacitance. Here, the input power to the plasma $P$ can be expressed by the following equation;

$$
P=2 k f W_{P}=2 k f\left(\frac{R_{P}}{R_{0}+R_{P}}\right) \frac{1}{2} C_{q} V_{\mathrm{BD}}{ }^{2}
$$

where $k$ is a number of discharges per half period of the applied voltage, $R_{0}$ is the resistance of the circuit, $R_{p}$ is the plasma resistance, $C_{q}$ is the capacitance of the quenching capacitor, $V_{\mathrm{BD}}$ is the breakdown voltage. It is clearly seen from Fig. 2 that the input power at $1 \mathrm{~mm}$ gap length increases discretely as the applied voltage increases of every $2 \mathrm{kV}_{\mathrm{pp}}$. These facts coincide with the

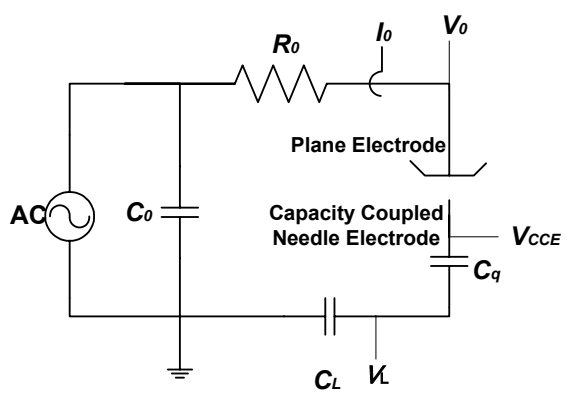

Fig. 1. Experimental setup for generation and measurement of capacity- coupled discharge $\left(R_{0}\right.$ : damping resistor, $C_{q}$ : quenching capacitor, $C_{L}$ : capacitor for $V-Q$ Lissajous measurement, $C_{0}$ : The capacitor for reducing the impedance of the power supply).

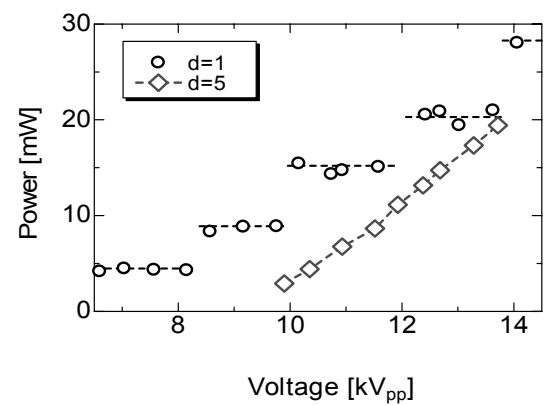

Fig. 2. Input power into the plasma as a function of applied voltage for two different gap lengths $\left(C_{q}=9.4 \mathrm{pF}\right)$

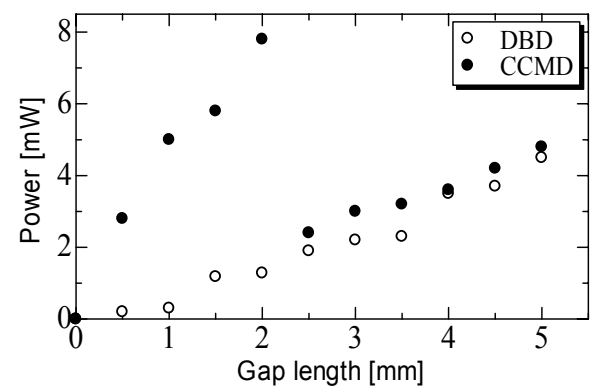

Fig. 3. Input power to the plasma as a function of gap length for two different quenching methods $\left(C_{q}=9.4 \mathrm{pF}\right)$

prediction from Eq. (1). The input power at $5 \mathrm{~mm}$ gap length increases linearly with increasing applied voltage. From the discharge current waveform measurement, an amount of the movement electric charge for one microdischarge at $5 \mathrm{~mm}$ gap length is much smaller than that at $1 \mathrm{~mm}$ gap length. The value of the amount charge of CCMD at $5 \mathrm{~mm}$ gap length is similar to that of conventional dielectric barrier discharge (DBD).

Figure 3 shows the input power into the discharge as a function of the gap length for two different discharge conditions; CCMD and DBD. The applied voltage was set to CCMD ignition voltage for each gap length $(k=1)$. The capacitance of the quenching capacitor for CCMD is $9.4 \mathrm{pF}$. The $2 \mathrm{~mm}$-thickness soda glass (7.5 in relative permittivity) plane for DBD is employed as dielectric barrier and the glass plane is set on the high voltage plane electrode. Figure 3 shows that the input power of DBD increases linearly with gap length. In the other, a discontinuous change is confirmed between the gap lengths of 2.0 and $2.5 \mathrm{~mm}$ of CCMD. This discontinuity means the transition of the discharge from the spark-like to the corona-like. The input power of CCMD is almost 5-10 times larger than that of DBD at shorter gap length than 2.5 $\mathrm{mm}$, whereas the input power of CCMD almost agrees with that of the DBD at larger gap length than $2.5 \mathrm{~mm}$. 


\section{Characteristics of Capacity Coupled Discharge in Atmospheric Pressure Air}

$\begin{array}{ll}\text { Tadahiro Sasaki* } & \text { Student Member } \\ \text { Reina Omukai** } & \text { Non-member } \\ \text { Seiji Mukaigawa* } & \text { Member } \\ \text { Koichi Takaki* }^{*} & \text { Member } \\ \text { Tamiya Fujiwara* } & \text { Member } \\ \text { Hiroshi Mase } * * & \text { Member } \\ \text { Noriyoshi Sato**** } & \text { Member }\end{array}$

This paper describes characteristics of capacity coupled discharge in atmospheric pressure air with focusing influence of gap length of point-to-plane electrode configuration on input power into the discharge. The discharge can be quenched in short time duration by inserting a small capacitance capacitor between the electrode and the ground. We employed a needle electrode and a coaxial cable as the quenching capacitor. The discharge was successfully quenched within $25 \mathrm{~ns}$ in duration according to $9.4 \mathrm{pF}$ in a capacitance of the quenching capacitor. The discharge was classified as two modes; a spark mode and a corona mode. At the spark mode, the power consumed in the discharge plasma was almost 10 times as large as that of a conventional dielectric barrier discharge. At the corona mode, the consumed energy was almost same value with that of the dielectric barrier discharge. A velocity of the discharge development was obtained to be $3 \times 10^{5} \mathrm{~m} / \mathrm{s}$ by an optical measurement.

Keywords : capacity-coupled multi-discharge, dielectric barrier discharge, power, streamer

\section{Introduction}

Research on atmospheric pressure nonthermal plasmas is motivated by various industrial applications; ozone generation, surface modification, material cleaning, decomposition of hazardous pollutants, light sources, etc ${ }^{(1)-(3)}$. A dielectric barrier discharge (DBD) is one of the atmospheric pressure plasma generation methods developed by Siemens as an ozonizer ${ }^{(4)}$. DBD is a typical ac discharge for the production of nonequilibrium thermal plasma at high gas pressure. DBD can occur between two electrodes with narrow gaps of 1 to $5 \mathrm{~mm}$, at least one of which should be covered with dielectric, when an ac voltage is applied to the electrodes. It limits the electric charge transported in the discharge, i.e., limits the current flow to the system and therefore, prevents the thermalization of the plasma.

Capacity-coupled multi-discharge (CCMD) has recently been proposed as a large-scale, atmospheric-pressure, dense plasma source $^{(5)}$. The discharge gaps in the CCMD consist of a common electrode and a number of compact electrodes which are directly coupled with small capacitors for quenching the discharge in a manner similar to the DBD. The CCMD is predicted to have interesting features for industrial applications; it is easy to control the plasma spatially because the discharge consists of only volume discharges without surface discharge; it is easy to control the energy deposited into the discharge plasma by changing the

* Iwate University

4-3-5, Ueda, Morioka 020-8551

** Tohoku Electric Power Co., Inc

1-7-1, Hontyou, Aoba, Sendai 980-8550

*** Ibaraki University

5-5-3, Takesuzu, Hitachi 317-0066

**** Tohoku University

4-17-113, Kadan, Aoba, Sendai 980-0815 quenching capacitor ${ }^{(6)}$. The self-quenching discharges coupled with small capacitors were employed for other objectives such as UV sources for photoionization of gas lasers ${ }^{(7)}$. However, this is the first case where the self-quenching discharges were used as nonthermal plasma sources.

Experiments have been carried out to understand the behavior of the CCMD. Mase et al. produced multi-discharge using twenty co-axial cables $20 \mathrm{~cm}$ in length ( $20 \mathrm{pF}$ for one cable). The results of these experiments showed that the amount of charge transfer for the 20 multi-discharge was $1.4 \mu \mathrm{C}$, which corresponded to $70 \mathrm{nC}$ charge transfer for a single discharge. The value was 10-100-fold that of the conventional $\mathrm{DBD}^{(5)}$. In general, ac voltage power supplies were used to generate the CCMD. Mase et al. also reported that the number of discharges per one ac applied voltage cycle can be controlled by the amplitude of the voltage. The number of discharges per half-period of ac applied voltage was successfully controlled from 1 to 4 by increasing the voltage ${ }^{(8)}$. Takaki et al. reported that the discharge was quenching in short time around $10 \mathrm{~ns}^{(6)}$.

In order to use the CCMD for various applications, it is necesarry to understand characteristics of the discharge more clearly such as influence of the electrode configuration on amout of the movement electric charge and consumed power. This paper describes characteristics of capacity coupled discharge in atmospheric pressure air with forcusing influence of gap length of point-to-plane electrode configuration on input power into the discharge. Furthermore, a velocity of the discharge propagation in the gap was obtained by an optical measurement using a photo-multiplier tube in order to compare with that of a typical streamer discharge. 


\section{Experimental Procedure}

Figure 1 shows the experimental setup of a self-quenching discharge system. The needle-to-plane electrode configuration was employed to fix the plasma position. A brass plane having $8 \mathrm{~cm}$ in diameter was used as a high voltage electrode. The needle electrode, which was $1.0 \mathrm{~cm}$ in length and $50 \mu \mathrm{m}$ in tip radius, was connected to the quenching capacitor. A coaxial cable RG58/U (53.5 $\Omega$ impedance, $94 \mathrm{pF} / \mathrm{m}$ capacitance, $268 \mathrm{nH} / \mathrm{m}$ inductance) was used as the capacitor for quenching the discharge. The capacitance of the quenching capacitor can be changed by the cable length at a rate of $94 \mathrm{pF} / \mathrm{m}$. The capacitance used in the experiment was $9.4 \mathrm{pF}$ according to a $10 \mathrm{~cm}$ length. A damping resistor $R_{0}$ was employed to reduce the inductance and capacitance (LC) resonant oscillation due to low impedance of the discharge plasma after breakdown. A typical value of the resistance $R_{0}$ was $50 \mathrm{k} \Omega$. The gap length between the needle tip and the plane electrode surface was changed from 1 to $5 \mathrm{~mm}$. The discharge gap was placed in atmospheric air.

The commercial ac voltage was raised to $1: 150$ by a leakage flux transformer $(15 \mathrm{~mA}, 50 \mathrm{~Hz})$. The raised voltage was $40 \mathrm{kV}$ in maximum peak-to-peak value, and it was applied to the plane electrode. The applied voltage was controlled by a variable transformer (slidac). To make the impedance of the power supply low and to realize the fast rise of the discharge current pulse, a 5.6 $\mathrm{nF}$ capacitor $C_{0}$ was connected in parallel with the power supply.

The potential of both electrodes was measured using high-voltage probes (SONY Tektronix P6015A). The discharge current was measured using a current monitor (PEASON CURRENT MONITOR 2877). The current and voltage signals were recorded with a digital storage oscilloscope (SONY Tektronix TDS 3054B). The energy deposited into the discharge plasma was obtained from voltage-charge curves $(V-Q$ Lissajous diagrams $)^{(9)}$. Previously, the energies obtained from $V$ - $Q$ Lissajous diagram were confirmed to be correct by comparison with the values calculated from the time integral of the product of the applied voltage and the current. In this study, we used mainly the $V-Q$ Lissajous diagram to measure the deposited energy. The electric charge was measured using a $0.05 \mu \mathrm{F}$ capacitor $C_{L}$ inserted between the quenching capacitor and the ground. The discharge development was measured optically using a side-on type Photo Multiplier Tube (Hamamatsu Photonics 1P28).

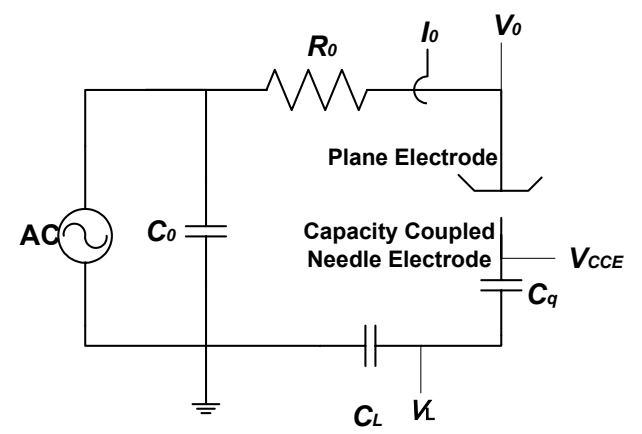

Fig. 1. Experimental setup for generation and measurement of capacity-coupled discharge $\left(R_{0}\right.$ : damping resistor, $C_{q}$ : quenching capacitor, $C_{L}$ : capacitor for $V-Q$ Lissajous measurement, $C_{0}$ : capacitor for reducing impedance of the power supply)

\section{Experiment Results}

3.1 Self-quenching Behavior One of special feature of the CCMD was expected to quench the discharge in short time. At first, we measured time-dependency of the discharge current and the electrode potentials. Figure 2 shows the time-dependency of the potentials of both electrodes at $1 \mathrm{~mm}$ gap length and $10.5 \mathrm{kV}_{\mathrm{pp}}$ applied voltage. In this case, we employed a $1.0 \mathrm{nF}$ quenching capacitor in order to reduce the decay of the quenching capacitor potential by a charge leakage from the quenching capacitor to the ground through the high-voltage probe. The damping resistor $R_{0}$ was also removed to decreasing influence of voltage drop caused by the circuit impedance and the discharge current. The applied voltage, i.e., the potential of the high-voltage electrode, shows an almost sinusoidal waveform, whereas the CCE potential changes stepwise. The voltage difference between steps is approximately $3 \mathrm{kV}$, while there is a scattering between 2.5 and $3.5 \mathrm{kV}$ due to the lack of a photo pre-ionization. The voltage difference between steps is considered to be close to the breakdown voltage $V_{\mathrm{BD}}{ }^{(5)}$. If we take $3 \mathrm{kV}$ as the breakdown voltage $V_{\mathrm{BD}}$, the integer $k$ is calculated as 3 at applied voltages of $10.5 \mathrm{kV}_{\mathrm{pp}}$, respectively, using following equation;

$$
k=\text { Integer Part of }\left(V_{\mathrm{PP}} / V_{\mathrm{BD}}\right),
$$

where $V_{\mathrm{PP}}$ is the ac potential in peak-to-peak value. These values show good agreement with the number of steps shown in Fig. 2.

Figure 3 shows that the time variation of discharge current and the both electrode potentials $V_{0}$ and $V_{\mathrm{CCE}}$ defined in Fig. 1 at $1 \mathrm{~mm}$ gap length and $10.0 \mathrm{kV}_{\mathrm{pp}}$ applied voltage. The capacitance of the quenching capacitor $C_{q}$ and the resistance of the damping resistor $R_{0}$ are $9.4 \mathrm{pF}$ and $50 \mathrm{k} \Omega$, respectively. The discharge occurred three times for each half cycle of the applied ac voltage as shown in Fig. 2. Figure 3 (a), (b) and (c) show the current and the potentials waveforms at first, second and third discharges, respectively. In all cases, when the discharge begins, the current increases from 0 to $3 \mathrm{~A}$ within a short time of about $10 \mathrm{~ns}$. This discharge current value is very large as compared with the current value (typically several tens of $\mathrm{mA}$ ) of one microdischarge of dielectric barrier discharge ${ }^{(10)}$. The discharge current charges the quenching capacitor and the CCE potential increases rapidly. The potential of the high-voltage electrode decreases simultaneously due to the impedance of the power source and the damping resistor $R_{0}$. As a result, the electric field strength between the electrodes decreases to almost zero at $10 \mathrm{~ns}$ after the discharge ignition, and the discharge is successfully quenched as predicted theoretically. This quenching time is close to that of $\mathrm{DBD}$, e.g., 50

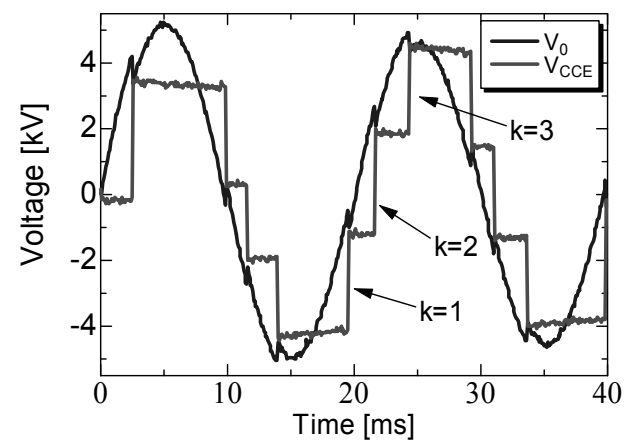

Fig. 2. Waveforms of drive electrode and capacity- coupled electrode potentials $\left(C_{q}=1.0 \mathrm{nF}, R_{0}=0 \Omega\right)$ 


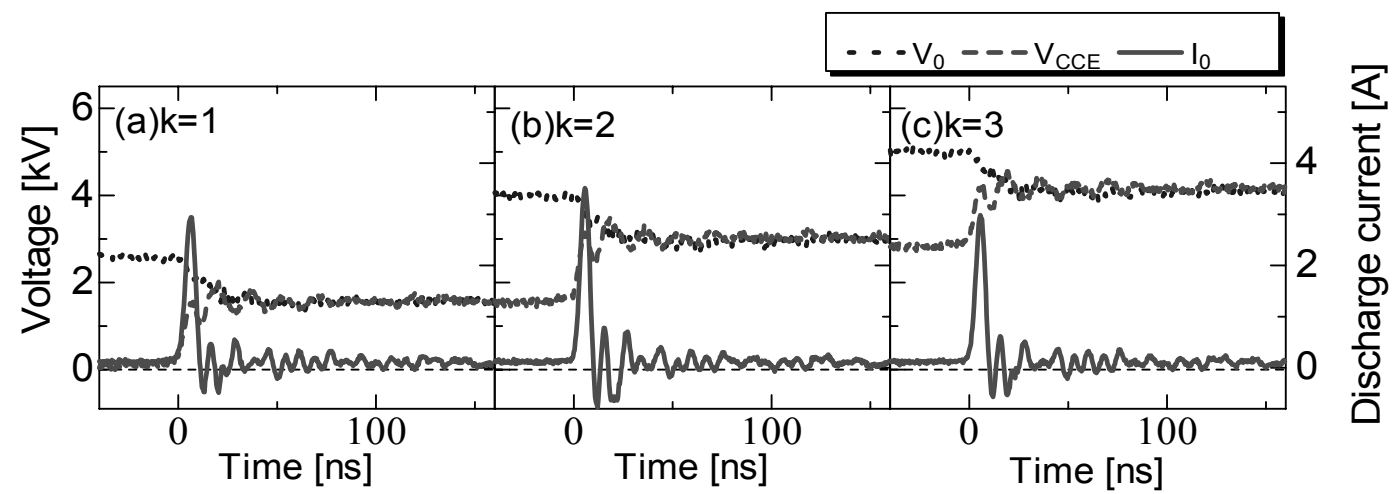

Fig. 3. Typical time-dependency of discharge current and electrode potentials ( $V_{\mathrm{CCE}}$ : potential of CCE, $V_{0}$ : potential of high voltage electrode, $\left.C_{q}=9.4 \mathrm{pF}, R_{0}=50 \mathrm{k} \Omega\right)$

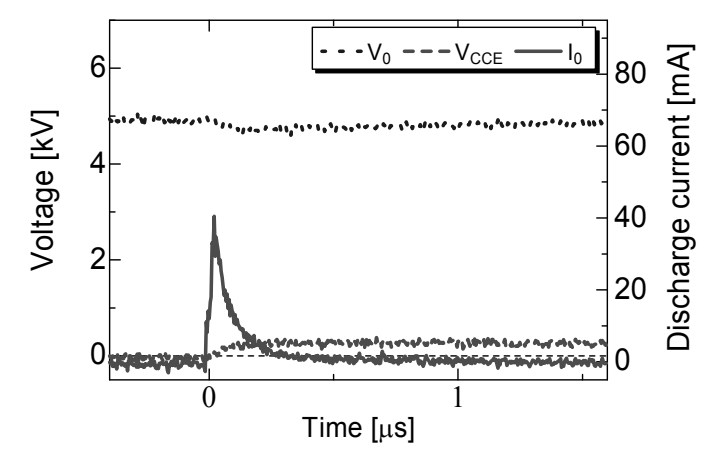

Fig. 4. Waveform of the discharge current and the electrode potentials at $5 \mathrm{~mm}$ gap length $\left(C_{q}=9.4 \mathrm{pF}, R_{0}=50 \mathrm{k} \Omega\right)$

ns as reported by Gibalov and Pietsch ${ }^{(10)}, 20 \mathrm{~ns}$ by Braun et al. ${ }^{(11)}$, and $20 \mathrm{~ns}$ from light emitted from discharge plasma reported by Dong et al. ${ }^{(12)}$.

3.2 Influence of Gap Length Figure 4 shows that the time variation of discharge current and the both electrode potentials $V_{0}$ and $V_{\mathrm{CCE}}$ at $5 \mathrm{~mm}$ gap length. Other conditions are same as shown in Fig. 3. In this case of $5 \mathrm{~mm}$ gap, the pulsive discharge current is also observed at $5 \mathrm{kV}$ of applied voltage $V_{0}$. The peak value of the discharge current is almost $40 \mathrm{~mA}$ which is much less than 3 A shown in Fig. 3. The amount of transfer charge for one discharge is roughly obtained to be $1.5 \mathrm{nC}$ by time-integrating the discharge current waveform. This value almost agrees with the calculating result using the CCE potential difference between before and after the discharge $\Delta V_{\mathrm{CCE}}$ as $C_{q} \times$ $\Delta V_{\mathrm{CCE}}(=9.4 \mathrm{pF} \times 150 \mathrm{~V})$. The $1.5 \mathrm{nC}$ amount of charge is almost one order less than the value $20 \mathrm{nC}$ at $1 \mathrm{~mm}$ gap length obtained using Fig. 3. Moreover, the voltage of the gap, $V_{0}-V_{\mathrm{CCE}}$, after the discharge is still larger than $4.5 \mathrm{kV}$ at $5 \mathrm{~mm}$ gap length, whereas the gap voltage after the discharge is almost zero at $1 \mathrm{~mm}$ gap length as shown in Fig. 3. This result reveals that the impedance of the discharge is still high and the ionization occurs spatial-partially around the tip of the needle electrode because of highly distorted strong electric field. Generally, that feature of the partial ionizing discharge can be classified as a corona discharge $e^{(13)(14)}$. On the other, the impedance of the discharge at 1 $\mathrm{mm}$ gap length is extremely small compared with that at $5 \mathrm{~mm}$ gap length. The feature of the low impedance pulsive discharge can be classified as a spark discharge; arc-like partially thermalized pulsive plasma ${ }^{(13)}$.

3.3 Input Power into the Discharge Figure 5 shows that the $V-Q$ Lissajous figure at $1 \mathrm{~mm}$ gap length and $10.0 \mathrm{kV}_{\mathrm{pp}}$

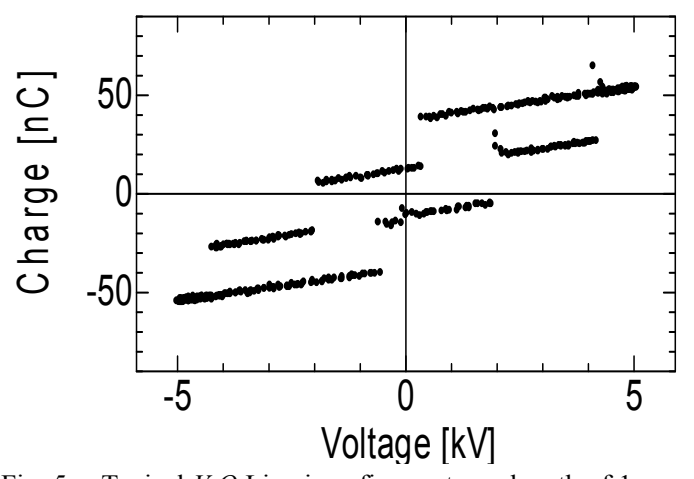

Fig. 5. Typical $V-Q$ Lissajous figure at gap length of $1 \mathrm{~mm}$ at $10 \mathrm{kV}_{\mathrm{pp}}$ applied voltage $\left(C_{q}=9.4 \mathrm{pF}, R_{0}=50 \mathrm{k} \Omega\right)$

applied voltage. The capacitance of the quenching capacitor is set to be $9.4 \mathrm{pF}$. The $V-Q$ Lissajous figure in Fig. 5 shows a discontinuously stepwise change. This stepwise change implies a large amount of the movement charge by one discharge as same manner with a glow mode $\mathrm{DBD}^{(15)}$. The discontinuous change in $V-Q$ Lissajous figure shows the generation of the discharge, and the number of the discharge per half-cycle of the applied voltage can be obtained to be three from Fig. 5. From the $V-Q$ Lissajous figure, the difference in electric charge between before and after discharges is almost constant at all discontinuously stepwise changes and is approximately $20 \mathrm{nC}$, which corresponds to $2.1 \mathrm{kV}$ in the potential difference of the CCE. This value of $20 \mathrm{nC}$ is about 10-100 times as large as the amount of the transfer electric charge (0.1-1 $\mathrm{nC})$ of the typical dielectric barrier discharge in the air at atmospheric pressure ${ }^{(2)}$.

Figure 6 shows that the $V-Q$ Lissajous figure at $5 \mathrm{~mm}$ gap length and $10.0 \mathrm{kV}_{\mathrm{pp}}$ applied voltage. The capacitance of the quenching capacitor is set to be $9.4 \mathrm{pF}$. The discontinuous change of the charge as shown in Fig. 5 at $1 \mathrm{~mm}$ gap length does not exist, and it is similar to $V-Q$ Lissajous figure in conventional DBD ${ }^{(16)}$. Figure 6 also shows that the amount of the movement charge by the discharge is almost $15 \mathrm{nC}$ per one half-cycle. The value of 15 $\mathrm{nC}$ is ten times larger than the $1.5 \mathrm{nC}$ of the one discharge transferred charge which is obtained with the current waveform shown in Fig. 4. This result indicates that a large number (more than 10) of discharges generates during a half-cycle of applied voltage as same manner with DBD.

Figure 7 shows the input power to the plasma as a function of the applied voltage for two different gap lengths at $9.4 \mathrm{pF}$ quenching capacitor capacitance. The applied voltage is changed from the breakdown voltage to $14 \mathrm{kV}_{\mathrm{pp}}$. The input powers were 
determined using $V-Q$ Lissajous figure as shown in Figss. 5 and 6. The discharge power at $1 \mathrm{~mm}$ gap was calculated using by dividing the $V-Q$ Lissajous figure shown in Fig. 5 into small rectangular pieces and summing the areas of all pieces ${ }^{(6)}$. Here, the input power to the plasma $P$ can be expressed by the following equation $^{(5)}$;

$$
P=2 k f W_{P}=2 k f\left(\frac{R_{P}}{R_{0}+R_{P}}\right) \frac{1}{2} C_{q} V_{\mathrm{BD}}{ }^{2},
$$

where $k$ is a number of discharges per half period of the applied voltage, $R_{0}$ is the resistance of the circuit, $R_{p}$ is the plasma resistance, $C_{q}$ is the capacitance of the quenching capacitor, $V_{\mathrm{BD}}$ is the breakdown voltage. Equation (2) implies that the input power $P$ changes discretely under these experimental conditions, because all values of Eq. (2) are nearly constant except $k$, and the value of $k$ can only be an integer. It is clearly seen from Fig. 7 that the input power increases discretely as the applied voltage increases of every $2 \mathrm{kV}_{\mathrm{pp}}$. Moreover, the value of the input power increases about every $5 \mathrm{~mW}$, and increases to an integer number $k$. These facts agree with the prediction from Eq. (2).

The input power at $5 \mathrm{~mm}$ gap length increases linearly with increasing applied voltage. The amount of the movement electric charge for one microdischarge at $5 \mathrm{~mm}$ gap length is much smaller than that at $1 \mathrm{~mm}$ gap length as mentioned in Sec. 3.2. This behavior of the $5 \mathrm{~mm}$ gap discharge is similar to that of DBD. The input power of the DBD can be expressed by the following equation;

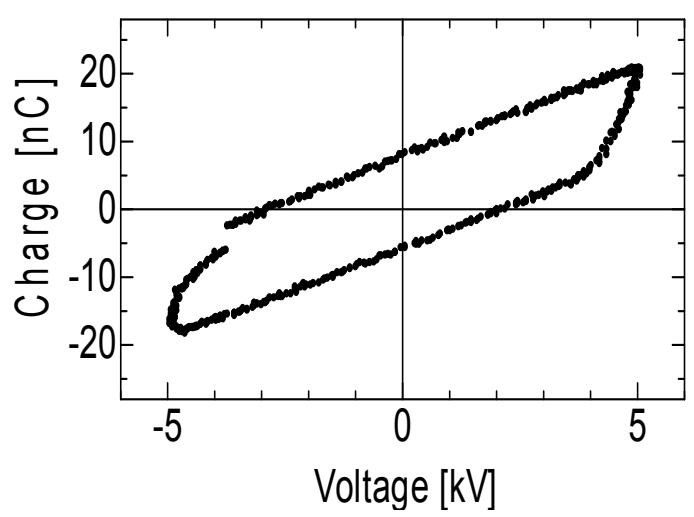

Fig. 6. Typical $V-Q$ Lissajous figure at gap length of $5 \mathrm{~mm}$ at $10 \mathrm{kV}_{\mathrm{pp}}$ applied voltage $\left(C_{q}=9.4 \mathrm{pF}, R_{0}=50 \mathrm{k} \Omega\right)$

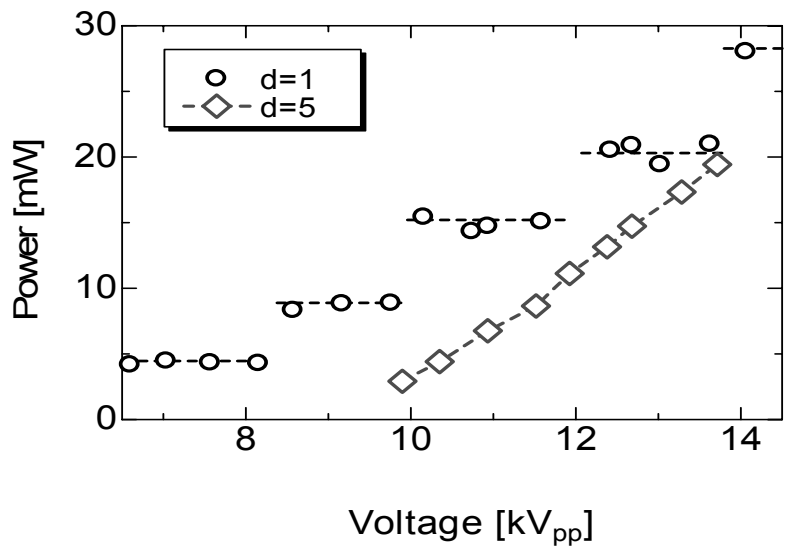

Fig. 7. Input power into the plasma as a function of applied voltage for two different gap lengths $\left(C_{q}=9.4 \mathrm{pF}, R_{0}=50 \mathrm{k} \Omega\right)$

$$
P_{\mathrm{DBD}}=4 f C_{g} e_{0}\left(e_{m}-\frac{C_{a}}{C} e_{0}\right),
$$

where $C=C_{g} C_{a} /\left(C_{g}+C_{a}\right), C_{g}$ and $C_{a}$ are the capacitance of the dielectric and the capacitance of the air, respectively. $e_{0}$ is an applied voltage, $e_{m}$ is a voltage between electrodes when there is no charge on the dielectric surface ${ }^{(9)(16)}$. This equation predicts that the input power linearly increases with applied voltage $e_{0}$. Therefore, the behavior of the input power at $5 \mathrm{~mm}$ gap length is similar to that of DBD, is not similar to that predicted by Eq. (2).

\section{Discussion}

\subsection{Comparison with Dielectric Barrier Discharge}

Figure 8 shows typical $V$ - $Q$ Lissajous figure of dielectric barrier discharge with same electrode configurations as shown in Fig. 1. The gap length and applied voltage are $5 \mathrm{~mm}$ and $10.0 \mathrm{kV}_{\mathrm{pp}}$, respectively. The $2 \mathrm{~mm}$-thickness soda glass ( 7.5 in relative permittivity) plane is employed as dielectric barrier and the glass plane is set on the high voltage plane electrode. The low voltage needle electrode is connected to the capacitor $C_{L}$ for $V-Q$ Lissajous measurement. The shape of the $V-Q$ Lissajous figure of DBD shown in Fig. 8 is similar to that of CCMD at $5 \mathrm{~mm}$ gap length as shown in Fig. 6.

Figure 9 shows the input power into the discharge as a function of the gap length for two different discharge conditions; CCMD and DBD. The applied voltage was set to CCMD ignition voltage for each gap length $(k=1)$. The capacitance of the quenching capacitor for CCMD is set to be $9.4 \mathrm{pF}$. The experimental condition of DBD is the same as shown in Fig. 8. Figure 9 shows that the input power of DBD increases linearly with gap length. On the other, a discontinuous change is confirmed between the gap lengths of 2.0 and $2.5 \mathrm{~mm}$ of CCMD. This discontinuity means the transition of the discharge from the spark-like to the corona-like as mentioned in Sec. 3.2. The input power of CCMD is almost 5-10 times larger than that of DBD at shorter gap length than $2.5 \mathrm{~mm}$, whereas the input power of CCMD almost agrees with that of the DBD at larger gap length than $2.5 \mathrm{~mm}$. The parallel-plane geometry is normally used as electrodes in conventional DBD chamber. In this case, the electric field distribution between the plane electrodes is almost independent of the gap length. In the present experiment, we employed a needle-to-plane geometry as CCMD electrodes. In this case, the electric field is highly distorted especially around tip of needle

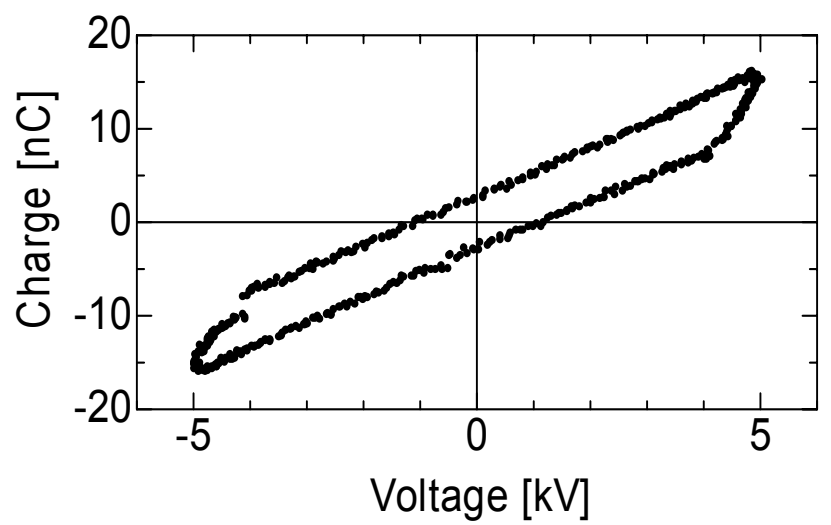

Fig. 8. Typical $V-Q$ Lissajous figure of $\mathrm{DBD}$ at $10 \mathrm{kV}_{\mathrm{pp}}$ applied voltage 


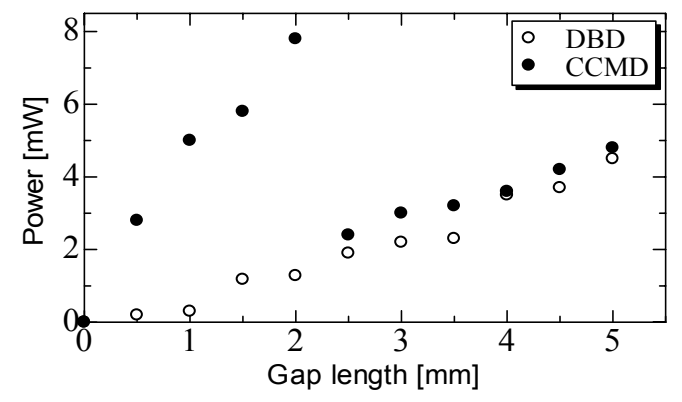

Fig. 9. Input power to the plasma as a function of gap length for two different quenching methods $\left(C_{q}=9.4 \mathrm{pF}, R_{0}=50 \mathrm{k} \Omega\right)$

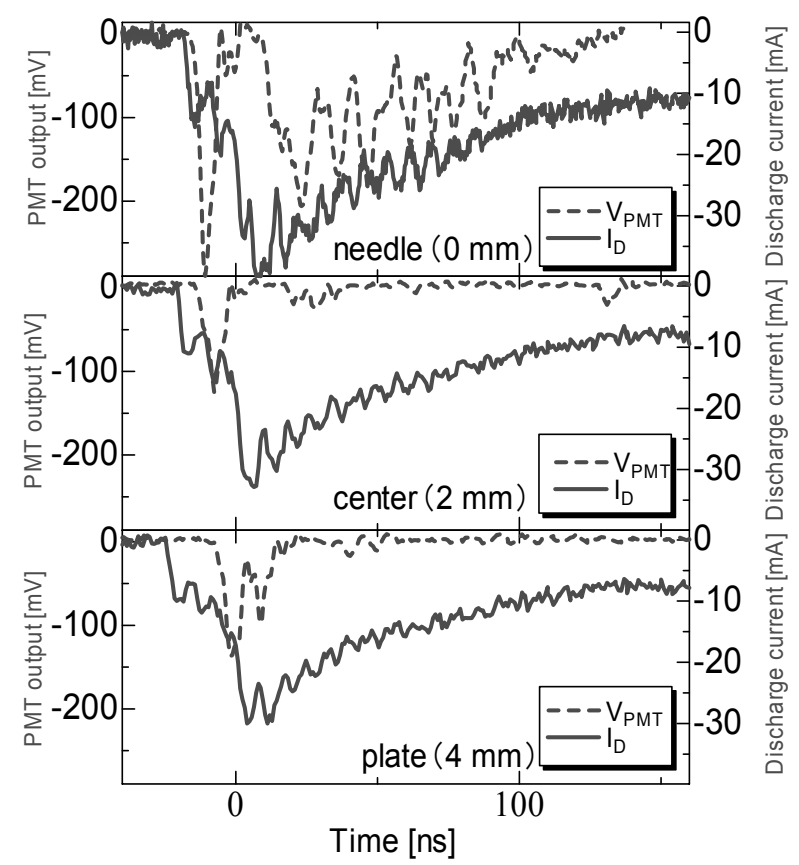

Fig. 10. CCMD discharge current waveforms and PMT outputs for three different measuring positions $\left(C_{q}=9.4 \mathrm{pF}, V_{0}\right.$ $\left.=10.5 \mathrm{kV}_{\mathrm{pp}}, d=5 \mathrm{~mm}\right)$

electrode $^{(17)}$. The change of the gap length strongly affects the electric field distribution between the electrodes and also affects the discharge properties ${ }^{(18)}$. This dependency of the gap length on the electric field distribution is one of the reasons for the drastic input power change between 2.0 and $2.5 \mathrm{~mm}$ in gap length.

4.2 Velocity of Discharge Development A velocity of the discharge development i.e. propagation speed of an ionizing wave can be measured using photo-multiplier tubes $(\mathrm{PMT})^{(19)(20)}$. Figure 10 shows that the CCMD current waveforms and the outputs of PMT from three different measuring positions of 0,2 and $4 \mathrm{~mm}$ from the tip of the low voltage needle electrode. The gap length and the capacitance of quenching capacitor were set to be $5 \mathrm{~mm}$ and $9.4 \mathrm{pF}$, respectively. The applied voltage was 10.5 $\mathrm{kV}_{\mathrm{pp}}$. The PMT output start to increase at only $2 \mathrm{~ns}$ after the discharge current start to increase at measuring point near the needle tip ( $0 \mathrm{~mm}$ position). However, the time deference between the current and PMT output increases with increasing the distance of measuring position from the needle tip.

Figure 11 shows the time difference from discharge current start to PMT signal as a function of distance from needle tip to PMT measuring position. The experimental condition is same as shown

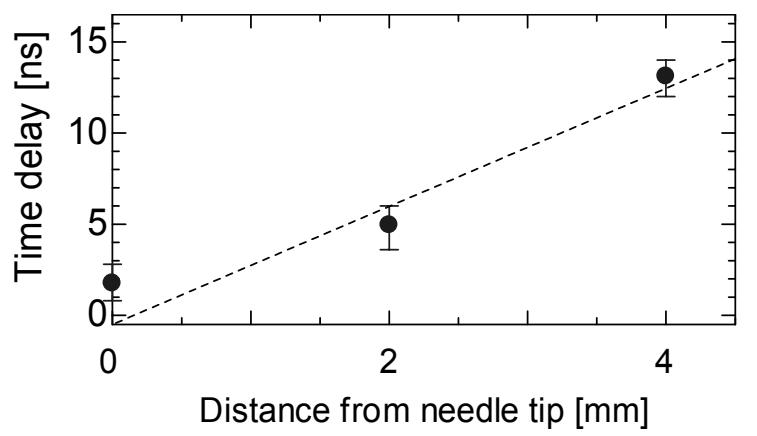

Fig. 11. Time difference from discharge current start to PMT output as a function of distance of PMT measuring point from needle tip $\left(C_{q}=9.4 \mathrm{pF}, V_{0}=10.5 \mathrm{kV}_{\mathrm{pp}}, d=5 \mathrm{~mm}\right)$

in Fig. 10. The each data was obtained by averaging via ten time measurements in order to reduce the scattering error. The velocity of the discharge development is roughly obtained to be $3 \times 10^{5} \mathrm{~m} / \mathrm{s}$ from Fig. 11. This value almost agrees with a streamer development speed in atmospheric pressure air ${ }^{(20)}$.

\section{Conclusions}

The characteristics of capacity coupled discharge in atmospheric pressure air were investigated with focusing influence of gap length of point-to-plane electrode configuration on input power into the discharge. The discharge was successfully quenched within $25 \mathrm{~ns}$ in duration according to $9.4 \mathrm{pF}$ in a capacitance of the quenching capacitor. The discharge was classified as two modes; a spark mode and a corona mode. At the spark mode, the power consumed in the discharge plasma was almost 10 times as large as that of a conventional dielectric barrier discharge. At the corona mode, the consumed energy was almost same value with that of the dielectric barrier discharge. A velocity of the discharge development was obtained to be $3 \times 10^{5} \mathrm{~m} / \mathrm{s}$ by an optical measurement.

\section{Acknowledgments}

The authors thank S. Okazaki, Professor Emeritus of Sophia University, for her valuable discussion and comments. The authors thank N. Sato, Professor Emeritus of Iwate University, for his cooperation. This work was supported by a Grants-in-Aid for Scientific Research (JSPS Fellowship No. 1803001)

(Manuscript received July 10, 2006, revised Dec. 4, 2006)

\section{References}

(1) B. Eliasson and U. Kogelschatz : "Air Pollution Control by Electrical Discharge", IEEE Trans. Plasma Sci., Vol.19, No.6, pp.1063-1076 (1991)

(2) X. Xu : "Dierectric barrier discharge properties and applications", Thin Solid Films, Vol.390, pp.237-242 (2001)

(3) B. Eliasson, M. Hirth, and U. Kogelschatz : "UV Excimer Radiation from Dierectric Barrier Discharges”, J. Phys. D: Appl. Phys., Vol.46, pp.299-303 (1987)

(4) K. H. Becker, R. J. Barker, and K. H. Schoencach : Non-Equilibrium Air Plasmas at Atmospheric Pressure, p.450, ISBN:0-7503-0962-8, IOP (2004)

(5) H. Mase, T. Fujiwara, and N. Sato : "Capacity-coupled multidischarge for atmospheric plasma production”, Appl. Phys. Lett., Vol.83, pp.5392-5394 (2003)

(6) K. Takaki, R. Ohmukai, S. Mukaigawa, T. Fujiwara, T. Miura, H. Mase, and N. Sato : "Energy Control of Capacity-Coupled Discharge in Atmospheric Air", Jpn. J. Appl. Phys., Vol.45, pp.1797-1800 (2006)

(7) P. R. Pearson and H. M. Lamberton : "Atmospheric Pressure $\mathrm{CO}_{2}$ Lasers Giving High Output Energy Per Unit Volume", IEEE Trans. Electronics. Sci., Vol.8, pp.145-149 (1972)

(8) H. Mase, T. Fujiwara, and N. Sato : "Capacity-Coupled Multidischarge at 
Atmospheric Pressure", IEEE Trans. Plasma Sci., Vol.32, pp.380-383 (2004)

(9) T. C. Manley : "The Electric Characteristics of the Ozonator Discharge", Trans. Electrochem. Soc., Vol.12, pp.83-96 (1943)

(10) V. I. Gibalov and G. J. Pietsch : "The development of dielectric barrier discharge in gas gaps and surfaces", J. Phys. D: Appl. Phys., Vol.33, pp.2618-2636 (2003)

(11) D. Braun, U. Kuchler, and G. J. Pietsch : "Microdischarges in air-fed ozonizers", J. Phys. D: Appl. Phys., Vol.24, pp.564-572 (1991)

(12) L. Dong, Z. Yin, X. Li, and L. Wang : "Spatio-temporal dynamics of discharge domains in a dielectric barrier discharge device", Plasma Sources Sci. Technol., Vol.12, pp.380-388 (2003)

(13) J. S. Chang, P. A. Lawless, and T. Yamamoto : "Corona Discharge Processes”, IEEE Trans. Plasma Sci., Vol.19, pp.1152-1166 (1991)

(14) B. Eliasson and U. Kogelschatz : "Nonequilibrium Volume Plasma Chemical Processing", IEEE Trans. Plasma Sci., Vol.19, pp.1063-1077 (1991)

(15) S. Okazaki, M. Kogoma, M. Uehara, and Y. Kimura : "Appearance of stable glow discharge in air, argon, oxygen and nitrogen at atmospheric pressure using a 50 Hz souce", J. Phys . D: Appl. Phys., Vol.26, pp.889-892 (1993)

(16) H. -E. Wargner, R. Brandenburg, K. V. Kozlov, A. Sonnenfeld, P. Michel, and J.F. Behnke : "The barrier discharge: basic properities and applications to surface treatment", Vacuum, Vol.71, pp.417-436 (2003)

(17) K. Takaki, M. Shimizu, S. Mukaigawa, and T. Fujiwara : "Effect of Electrode Shape in Dielectric Barrier Discharge Plasma Reactor for NOx Removal", IEEE Trans. Plasma Sci., Vol.32, No.1, pp.32-38 (2004)

(18) M. A. Jani, K. Toda, K. Takaki, and T. Fujiwara : "An experimental comparison between electrode shaps for NOx treatment using a dielectric barrier discharge", J. Phys. D: Appl. Phys., Vol.33, pp.3078-3082 (2000)

(19) T. Fujiwara, T. Shimada, and K. Sugita : "Development of the primary electron avalanche under an induced toroidal electric field in nitrogen", $J$. Phys. D: Appl. Phys., Vol.16, pp.1217-1224 (1983)

(20) N. L. Allen and A. Ghaffar : "The conditions required for the propagation of a cathode-directed positive streamer in air", J. Phys. D: Appl. Phys., Vol.28, pp.331-337 (1995)

Tadahiro Sasaki

(Student Member) was born in Iwate, Japan, on March

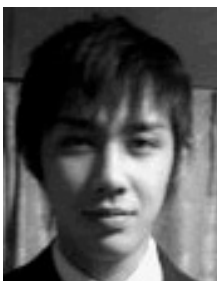

8, 1984. He received the B.E. degrees in electrical engineering from Iwate University, Japan, in 2006. He is now in graduate course in electrical engineering of Iwate University, Japan. His current interests include generation of discharge plasma at atmospheric pressure.

Reina Omukai

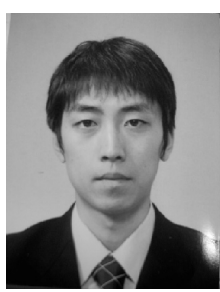

(Non-member) was born in Totigi, Japan, on August 25, 1981. He received the B.E. and M.E. degrees in electrical engineering from Iwate University, Japan, in 2004 and 2006, respectively. In 2006, he was in the Tohoku Electric Power Co., Inc., Japan.

Seiji Mukaigawa

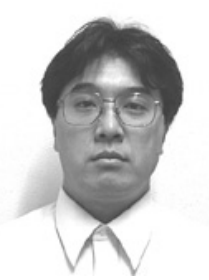

(Member) received the B. Sci. degree in physics from Ibaraki University, Mito, Janan, in 1993, and M. Sci. and Dr. Sci. degrees in physics from Hiroshima University, Higashi-Hiroshima, Japan, in 1995 and 1998, respectively. In 1998 and 2001, he was a Post doctoral Fellowship at Research center of nanodevices and systems in Hiroshima University. In 2001, he was a Research Associate with the Department of Electrical and Electronics Engineering, Iwate University, Morioka City, Japan.
Koichi Takaki

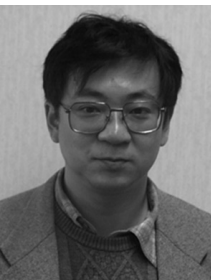

(Member) was born in Fukuoka, Japan, on October 16, 1963. He received the B. Eng., M. Eng. and Dr. Eng. degrees in electrical engineering from Kumamoto University, Kumamoto, Japan, in 1986, 1988 and 1995, respectively. In 1989 and 1993, he was a Research Associate and Lecturer, respectively, at Oita National College of Technology. In 1996 and 2000, he was a Research Associate and Associate Professor, respectively, with the Department of Electrical and Electronics Engineering, Iwate University, Morioka City, Japan. During 2000-2001, he was a Visiting Scientist at the McMaster University, Hamilton, ONT, Canada. His current interests are basic and applied research in various aspects of gaseous discharge and pulsed power.

Tamiya Fujiwara

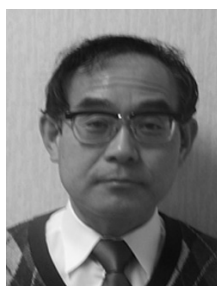

pressure.
(Member) received the B.E., M.E. and Dr. E. degrees in electrical engineering from Tohoku University, Sendai, Japan, in 1971, 1973, and 1986, respectively.From 1987 to 1988 , he was a visiting scientist at MIT, Cambridge, MA. In 1994 he became a Professor of Iwate University, Morioka, Japan. His research involves the phenomena and applications of gas discharge. His current interests include generation of discharge plasma at atmospheric

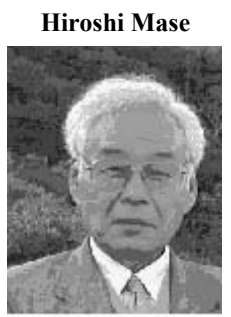

(Member) was born in Ibaraki, Japan, in 1936. He received $\mathrm{BE}, \mathrm{ME}$, and Dr. $\mathrm{E}$ degree from Tohoku University, Sendai, Japan, in 1958, 1962, and 1965, respectively. He was a Professor of Ibaraki University, Hitachi, Japan, from 1979 to 2001. He is now a Professor Emeritus, Ibaraki University. He worked on the low-pressure discharges, plasma physics and their applications; plasma productions for materials processing, rotating plasma, plasma centrifuge, formation of fullerenes, etc. Prof. Mase is a member of The Japan Society of Plasma Science and Nuclear Fusion Research, The Physical Society of Japan and American Physical Society.

Noriyoshi Sato

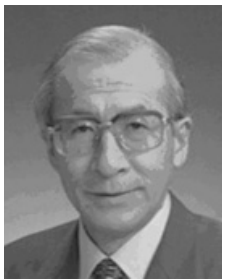

(Member) was born in Miyagi, Japan, in 1938. He received B.E., M.E., and Dr.E degree from Tohoku University, Sendai, Japan, in 1960, 1962 and 1965, respectively. He was a Professor in the Graduate School of Engineering, Tohoku University from 1979 to 2001. He is now a Professor Emeritus, Tohoku University. His works cover a wide range of plasma physics and applications. Recent topics of his research are concerned with plasma potential formation and related phenomena, fullerene plasmas, fine-particle plasmas, active plasma control for various kinds of plasma applications. Prof. Sato is a member of The Japan Society of Plasma Science and Nuclear Fusion Research, The Physical Society of Japan and The Japan Society of Applied Physics. 